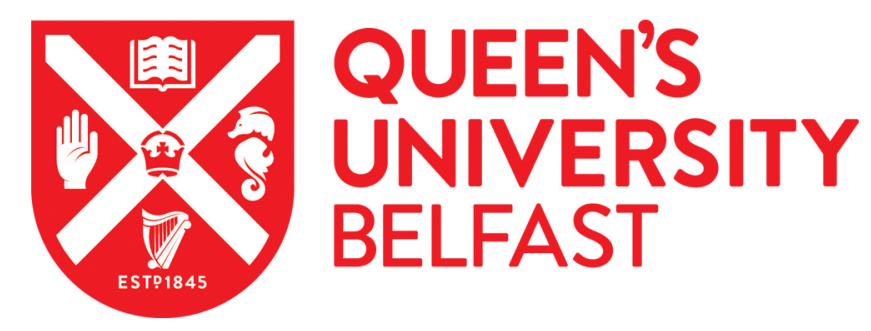

\title{
Phase curve and variability analysis of WASP-12b using TESS photometry
}

Owens, N., de Mooij, E. J. W., Watson, C. A., \& Hooton, M. J. (2021). Phase curve and variability analysis of WASP-12b using TESS photometry. Monthly Notices of the Royal Astronomical Society: Letters.

https://doi.org/10.1093/mnrasl/slab014

Published in:

Monthly Notices of the Royal Astronomical Society: Letters

Document Version:

Peer reviewed version

Queen's University Belfast - Research Portal:

Link to publication record in Queen's University Belfast Research Portal

Publisher rights

Copyright 2021 the Authors

This work is made available online in accordance with the publisher's policies. Please refer to any applicable terms of use of the publisher.

\section{General rights}

Copyright for the publications made accessible via the Queen's University Belfast Research Portal is retained by the author(s) and / or other copyright owners and it is a condition of accessing these publications that users recognise and abide by the legal requirements associated with these rights.

Take down policy

The Research Portal is Queen's institutional repository that provides access to Queen's research output. Every effort has been made to ensure that content in the Research Portal does not infringe any person's rights, or applicable UK laws. If you discover content in the Research Portal that you believe breaches copyright or violates any law, please contact openaccess@qub.ac.uk. 


\title{
Phase curve and variability analysis of WASP-12b using TESS photometry
}

\author{
Niall Owens, ${ }^{1 \star}$ E.J.W. de Mooij, ${ }^{1}$ C.A. Watson ${ }^{1}$ M.J. Hooton ${ }^{2}$ \\ ${ }^{1}$ Astrophysics Research Centre, Queen's University Belfast, Belfast BT7 1NN, UK \\ ${ }^{2}$ Physikalisches Institut, University of Bern, Gesellschaftsstr. 6, 3012 Bern, Switzerland
}

Accepted XXX. Received YYY; in original form ZZZ

\begin{abstract}
We analyse Sector 20 TESS photometry of the ultra-hot Jupiter WASP-12b, and extract its phase curve to study the planet's atmospheric properties. We successfully recover the phase curve with an amplitude of $549 \pm 62 \mathrm{ppm}$, and a secondary eclipse depth of $609_{-73}^{+74} \mathrm{ppm}$. The peak of the phase curve is shifted by $0.049 \pm 0.015$ in phase, implying that the brightest spot in the atmosphere is shifted from the substellar point towards the planet's evening terminator. Assuming zero albedo, the eclipse depth infers a day-side brightness temperature of $3128_{-68}^{+64} \mathrm{~K}$. No significant detection of flux from the night-side is found at $60 \pm 97 \mathrm{ppm}$, implying a nightside brightness temperature of $<2529 \mathrm{~K}(1-\sigma)$. We do not detect any significant variability in the light from the planet over the $\sim 27$ days of the TESS observations. Finally, we note that an ephemeris model taking orbital decay into account provides a significantly better fit than a constant-period model.
\end{abstract}

Key words: planets and satellites: individual: WASP- $12 \mathrm{~b}$ - planets and satellites: atmospheres - planets and satellites: gaseous - techniques: photometric

\section{INTRODUCTION}

The atmosphere of a transiting exoplanet can be characterised through observations of its secondary eclipse and phase curve. The depth of the secondary eclipse allows the planetary day-side emission to be measured, providing constraints on the day-side temperature of the atmospheric layers being probed. The day-night contrast can additionally be obtained from the phase curve, as well as effectively mapping the emission of the emitting atmospheric layer as a function of longitude (e.g. Knutson et al. 2007). Due to their very nature, hot Jupiters are well suited for this method of characterisation and it has been revealed that their bright spot is often offset in phase from the substellar point (e.g. Knutson et al. 2007; de Mooij \& Snellen 2009; Zhang et al. 2018). This allows the transport of energy within the atmosphere of the planet to be studied.

The spectral energy distribution (SED) for a hot Jupiter peaks in the infrared, making observations in this regime favourable due to the decreased contrast between the planet and its host star (e.g. Zellem et al. 2014; Bell et al. 2019). Due to the strong wavelengthdependence of the planetary flux at optical wavelengths, the contrast between the day- and night-side flux is greater than in the nearinfrared. At optical wavelengths, the night-side will appear almost completely dark (e.g Snellen et al. 2009). However the planet-star contrast for the day-side is also significantly lower, making detections more challenging. Furthermore, the flux observed in the optical

^ E-mail: nowens09@qub.ac.uk will constitute both thermally emitted- and reflected starlight that when combined with multi-wavelength studies - can effectively constrain the planet's geometric albedo. One very interesting advantage of this wavelength regime is that it is very sensitive to temperature changes within the layer of the atmosphere being probed, potentially revealing temporal-evolving features. This was the case in Armstrong et al. (2016) who observed the peak emission of the hot Jupiter HAT-P-7b shifting between the morning and evening hemispheres, which could be due to variable cloud coverage.

In this Letter we investigate the phase-curve of the ultra-hot Jupiter WASP-12b (Hebb et al. 2009), which orbits a G0-type star every 1.09 days. This makes it one of the most heavily irradiated exoplanets known, with an expected day-side temperature of $\sim 3,000 \mathrm{~K}$. Its extreme temperatures have allowed for its secondary eclipse to be observed throughout the infrared (Croll et al. 2011; Campo et al. 2011; Zhao et al. 2012; Crossfield et al. 2012; Cowan et al. 2012; Stevenson et al. 2014; Bell et al. 2019) as well as the optical (LópezMorales et al. 2010; Föhring et al. 2013; Hooton et al. 2019; von Essen et al. 2019). Additionally, the geometric albedo for WASP$12 \mathrm{~b}$ has been measured to be very low $\left(A_{\mathrm{g}}<0.064\right)$ at optical and near-ultraviolet wavelengths (Bell et al. 2017) suggesting the planet to be one of the darkest known to date. Of particular interest is the observed variability in reported secondary depths within the $z^{\prime}$-band; the secondary eclipse observed in López-Morales et al. (2010) and Föhring et al. (2013) were conducted just 2 months apart yet disagree in their reported depths by over $3 \sigma$. Further secondary eclipse depths reported by Hooton et al. (2019) in the $i^{\prime}$-band are 


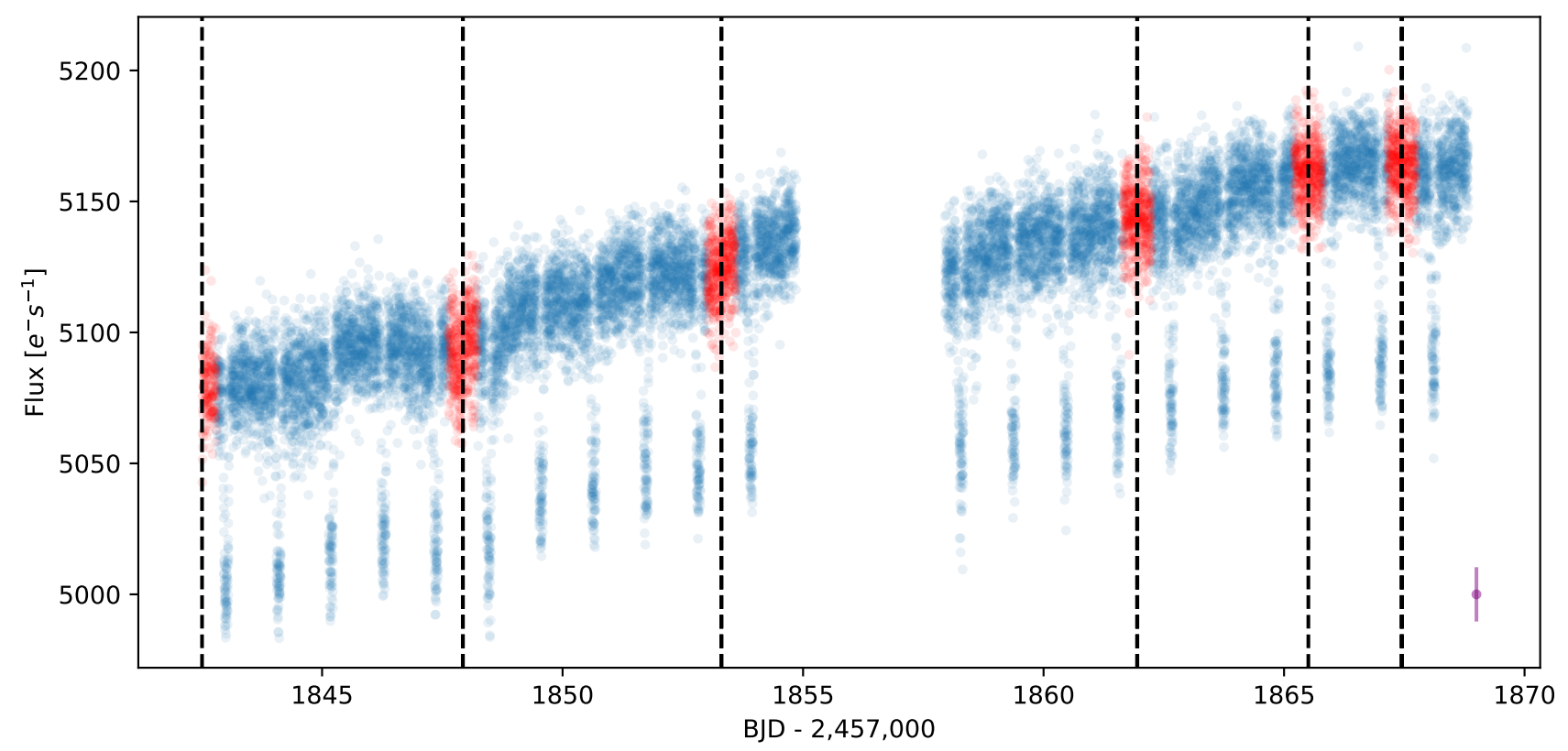

Figure 1. SAP light curve for WASP-12 with flagged points and NaNs removed. Black horizontal lines denote times that were identified as momentum dumps by the data quality flags. Discarded data are shaded in red, spanning 0.6 days centred on each momemtum dump. The gap present in the middle is due to a pause to enable data downlink. Clearly present are the 21 transits of WASP-12. A typical errorbar for this dataset is shown in the bottom-right corner.

also discrepant on the order of $3 \sigma$ and were conducted a year apart. Such a discrepancy corresponds to variations of around $400 \mathrm{~K}$ which may be the result of atmospheric variability, such as storms. Furthermore, WASP-12b marks the first planet where orbital decay due to tidal dissipation has been confidently observed (Yee et al. 2020), see also recent work by Turner et al. (2020).

Here we present the first full optical phase curve of WASP-12b as observed by TESS. In Section 2, we discuss the observations and preparation of the data for analysis; in Section 3 we outline our detrending strategy as well as our light curve model; in Section 4 we present our results and discuss their implications, including a search for variability within the data as well as orbital decay; and finally we present our conclusions in Section 5.

\section{TESS OBSERVATIONS}

The TESS spacecraft observed WASP-12 (TIC 86396382) at 2minute cadence during its Sector 20 observations from 2019 December 24 to 2020 January 20, over 2 full TESS orbits.

The 2-minute data collected by TESS is processed by the TESS Science Processing Operations Center (SPOC, Jenkins et al. 2016) and produces the data products that we use in this analysis. These files consist of both simple aperture photometry (SAP) and presearch data conditioning simple aperture photometry (PDCSAP) light curves. The SAP light curve is produced using an optimum aperture mask as determined by TESS. This data has been calibrated, background-subtracted, and has had cosmic ray events removed but has not been detrended or had any other systematic error correction techniques applied. The PDC light curve is treated similarly but has been thoroughly detrended using SPOC algorithms, and makes use of cotrending basis vectors (CBVs) to find common trends within the ensemble flux data that are then subsequently removed from the target flux-series.

Before fitting and performing correction for instrumental effects, all flagged and $\mathrm{NaN}$ points were removed. Flagged points also include reaction wheel desaturation events (momentum dumps). During these events, fine pointing is lost, compromising the quality of the data both leading up to- and after the event. This results in large discontinuities within the data about these points and any attempts to detrend such events prove to be challenging. We therefore decided to conservatively remove 0.6 days of data centred on each momentum dump due to the severe unpredictable ramp-like fluctuations that take place. Furthermore, the published $1 \sigma$ errors are found to be significantly overestimated due to an issue in the TESS analysis pipeline (TESS Science Support Center priv. comm.), so we opt to calculate the spread in the residuals between the data and best-fit model (see Sect. 3.1) and then scale the errors appropriately.

In total we analyse 14, 243 points, comprising 21 transits and 20 secondary eclipses. The lightcurve can be seen in Fig. 1.

\section{ANALYSIS}

\subsection{Light curve model}

The light curve model was generated using a near-identical model to that used by Esteves et al. $(2013,2015)$ where we assume a circular orbit. The model accounts for the transit $F_{\text {transit }}$, the secondary eclipse $F_{\text {ecl }}$, the phase-curve modulation $F_{\mathrm{p}}$, and the effect due to ellipsoidal distortions and Doppler beaming $F_{\mathrm{m}}$. Each term is a function of the planet's phase $\phi$, with transit and secondary eclipse at a phase of 0 and 0.5 , respectively. The entire light curve is modelled:

$F_{\text {transit }}(\phi) \cdot\left(F_{\mathrm{m}}(\phi)+1\right)+F_{\text {ecl }}(\phi)+F_{\mathrm{p}}(\phi+\theta)$ 
where $\theta$ is the brightness offset in phase from the planet's substellar point. We use the transit model $F_{\text {transit }}$ as formulated by Mandel \& Agol (2002) and use the Python package batman (Kreidberg 2015) to calculate the transit light curve for the WASP-12 system. Note that we use a quadratic limb-darkening law in these solutions, and in our fits we constrain the limb-darkening coefficients (LDCs) by fitting for $q_{1}$ and $q_{2}$ as advocated by Kipping (2013). From the transit we can determine the planet-to-star radius ratio $R_{\mathrm{p}} / R_{*}$, the scaled planetary semi-major axis $a / R_{*}$, and the orbital inclination $i$. While the period $P$ and midtransit time $t_{0}$ can be determined as well, we opt to use the ephemeris as described in Yee et al. (2020) as the short baseline of the TESS data would not allow the orbital decay of WASP- $12 b$ to be directly measured.

The secondary eclipse $F_{\text {ecl }}$ is modelled by normalising the transit solution assuming a uniformly bright source and scaling appropriately. The planet's apparent surface is modelled as a Lambert sphere and is described by:

$F_{\mathrm{p}}(\phi)=A_{\mathrm{p}} \frac{\sin z(\phi)+(\pi-z(\phi)) \cos z(\phi)}{\pi}$

where $A_{\mathrm{p}}$ is the peak brightness amplitude, and $z(\phi)$ is defined as:

$\cos z(\phi)=-\sin i \cos (2 \pi[\phi+\theta])$

The final parameter we model and fit is $F_{\mathrm{m}}$, which accounts for Doppler boosting of the system as well as ellipsoidal variations due to tidal effects on the star by the planet. As both of these effects are dependent upon the planetary mass $M_{p}$, they can both be expressed as a single equation:

$$
\begin{aligned}
F_{m} & =M_{p}\left\{\left(\frac{2 \pi G}{P}\right)^{1 / 3} \frac{\alpha_{d} \sin i}{c M_{*}^{2 / 3}} f_{d}-\frac{\alpha_{2} \sin ^{2} i}{M_{*}} f_{e}\right\} \\
f_{e} & =\left(\frac{a}{R_{*}}\right)^{-3} \cos (2 \cdot 2 \pi \phi)+\left(\frac{a}{R_{*}}\right)^{-4} f_{1} \cos (2 \pi \phi) \\
& +\left(\frac{a}{R_{*}}\right)^{-4} f_{2} \cos (3 \cdot 2 \pi \phi) \\
f_{d} & =\sin 2 \pi \phi
\end{aligned}
$$

where $G$ is the gravitational constant, $c$ is the speed of light, and $\alpha_{d}$ is the photon-weighted bandpass-integrated beaming factor. $\alpha_{d}$ is calculated as outlined in Loeb \& Gaudi (2003); Bloemen et al. (2011). $\alpha_{2}$ is a function of the limb- and gravity darkening coefficients within the TESS waveband for WASP-12 (Claret 2017). $f_{d}$ and $f_{e}$ describe the phase-dependent modulations for Doppler boosting and ellipsoidal variations, respectively, with $f_{1}$ and $f_{2}$ being constants determining the amplitude of the higher-order ellipsoidal terms and are dependent on the limb- and gravity darkening coefficients for the star. The calculation of these terms as well as that of $\alpha_{2}$ are outlined in Esteves et al. (2013). We note that $F_{\mathrm{m}}$ does not constitute part of the planetary phase curve but is due to the star influencing the overall light curve. While these features are detected, the planetary mass still remains unconstrained; we therefore opt to use Gaussian priors (see Table 1).

\subsection{Detrending strategy and joint-fit}

In detrending the data, we make use of CBVs to fit the long- and short term systematic trends within the data to minimise the impact other detrending techniques may have on the phase curve. The CBVs come ordered in terms of significance, and we progressively add more CBVs (of lower significance) and fit the data, calculating the

\begin{tabular}{|c|c|c|}
\hline Parameter & Prior & Best-fit \\
\hline \multicolumn{3}{|c|}{ Orbital parameters } \\
\hline$P$ (days) & fixed & $1.091420107(42)^{a}$ \\
\hline$t_{0}(\mathrm{BJD})$ & fixed & $2456305.455809(32)^{a}$ \\
\hline$d P / d N$ & fixed & $-10.04(69) \times 10^{-10} a$ \\
\hline$a / R_{*}$ & $\mathcal{N}(3.03,0.034)^{b}$ & $3.038 \pm 0.022$ \\
\hline$i$ (degrees) & $\mathcal{N}(83.37,0.7)^{b}$ & $83.21_{-0.48}^{+0.51}$ \\
\hline \multicolumn{3}{|c|}{ Transit parameters } \\
\hline$R_{\mathrm{p}} / R_{*}$ & - & $0.1174 \pm 0.0007$ \\
\hline$u_{1}$ & - & $0.225_{-0.119}^{+0.177}$ \\
\hline$u_{2}$ & - & $0.282_{-0.136}^{+0.194}$ \\
\hline \multicolumn{3}{|c|}{ Phase curve } \\
\hline$F_{\mathrm{ecl}}(\mathrm{ppm})$ & - & $609_{-73}^{+74}$ \\
\hline$A_{\mathrm{p}}(\mathrm{ppm})$ & - & $549 \pm 62$ \\
\hline$\theta$ (phase) & - & $0.049 \pm 0.015$ \\
\hline$M_{\mathrm{p}}\left(\mathrm{M}_{\mathrm{J}}\right)$ & $\mathcal{N}(1.47,0.076)^{b}$ & $1.48 \pm 0.08$ \\
\hline$F_{\mathrm{n}}(\mathrm{ppm})$ & - & $60 \pm 97$ \\
\hline \multicolumn{3}{|c|}{ Derived } \\
\hline$A_{\mathrm{g}, \mathrm{ecl}}$ & - & $0.408 \pm 0.050$ \\
\hline$T_{\mathrm{B}, \text { day }}(\mathrm{K})$ & - & $3128_{-68}^{+64}$ \\
\hline$T_{\mathrm{B}, \text { night }}(\mathrm{K})$ & - & $<2529(1-\sigma)$ \\
\hline$A_{\mathrm{g}, \max }$ & - & no solution \\
\hline$T_{\max }(\mathrm{K})$ & - & no solution \\
\hline$A_{\mathrm{g}, \mathrm{hom}}$ & - & $0.385_{-0.061}^{+0.060}$ \\
\hline$T_{\text {hom }}(\mathrm{K})$ & - & $2064_{-120}^{+105}$ \\
\hline$f_{\lim }$ & - & $0.556 \pm 0.047$ \\
\hline
\end{tabular}
BIC for each fit. We select the model that has the BIC minimised,
Table 1. Best-fit parameters, and derived parameters, from our MCMC fit for WASP-12b.

and this occurs when we include $9 \mathrm{CBV}$. We opt to fit this number of CBVs in addition to our phase curve model. We note that beyond $2 \mathrm{CBV}$ s, there is no significant difference in the derived planet's parameters.

The CBV function we utilise is of the form:

$C B V s=a_{0}+\sum_{k=1}^{8} a_{k} C_{k}(t)$

where $C$ represents a given CBV as a function of time $t$, and $a$ represents the coefficient or weighting given to that $\mathrm{CBV}$. The full model fit is therefore given as:

$\left[F_{\text {transit }}(\phi) \cdot\left(F_{\mathrm{m}}(\phi)+1\right)+F_{\text {ecl }}(\phi)+F_{\mathrm{p}}(\phi+\theta)\right] \times C B V s$

We perform our fit using emcee (Foreman-Mackey et al. 2013) and use 64 walkers and 15,625 steps for a total of 1,000,000 links with 29 free parameters. Table 1 outlines the Gaussian priors used.

For completeness, we also take the traditional approach of 
fitting a polynomial to each orbit to remove slow trends, and additionally present the PDCSAP flux. Both analyses can be found in Appendix A.

\section{DISCUSSION}

The results of our MCMC fit are presented in Table 1 and Figure 2. In addition, we performed a residual permutation bootstrap analysis to investigate the impact of correlated noise on the uncertainties. We found that this resulted in smaller uncertainties on the best fit parameters, indicating that the impact of systematic effects was minor. We therefore adopt the uncertainties from the MCMC fit. The results of the fits to each of the individual TESS orbits are provided and compared in Appendix A, we see no significant differences between the key parameters.

\subsection{Brightness temperatures, and geometric albedos}

We can calculate the geometric albedo for WASP-12b if we assume no thermal contribution:

$F_{\text {ecl }}=A_{\mathrm{g}, \mathrm{ecl}}\left(\frac{R_{\mathrm{p}}}{a}\right)^{2}$.

We find for WASP-12 that $A_{\mathrm{g}, \text { ecl }}<0.458(1 \sigma)$. If we assume a bond albedo $A_{\mathrm{B}}$ of 0 , then we can determine the planet's equilibrium temperature - which assumes no reflective component - to be (López-Morales \& Seager 2007):

$T_{\text {eq }}=T_{*}\left(\frac{a}{R_{*}}\right)^{-1 / 2} f^{1 / 4}$

where $f$ is a factor that defines the two limiting cases: homogeneous re-distribution $(f=1 / 4)$ designated $T_{\text {eq, hom, }}$, and instant re-radiation $(f=2 / 3)$ as $T_{\text {eq, } m a x}$, and $T_{*}$ and $R_{*}$ are the stellar temperature and radius, respectively. These cases are indicative of the efficiency of the atmosphere to absorb and distribute the incoming radiation, and is dependent on the composition of the planetary atmosphere. For WASP-12b, we find $T_{\text {eq, } \max }=3275 \mathrm{~K}$, and $T_{\text {eq, hom }}$ $=2560 \mathrm{~K}$. In addition, we calculate the brightness temperatures for both the day- and night-side of WASP-12b, following Esteves et al. (2013). We take the TESS bandpass into account, and use stellar models from the BT-Settl grid (Baraffe et al. 2015) for stellar parameters matching that of WASP-12 as determined by Delgado Mena et al. (2015). These brightness temperatures can be found in Tab. 1. We note that, given the bandpass of TESS, the eclipse depth will be a combination of both reflected light and thermal emission. As in Esteves et al. (2013), we try to self-consistently solve for the geometric albedo by assuming isotropic scattering $\left(\mathrm{A}_{\mathrm{B}}=\frac{3}{2} A_{\mathrm{g}}\right)$ for two limiting cases in terms of heat distribution throughout the atmosphere, homogeneous redistribution and instant re-radiation.

For a homogeneous temperature the day-side eclipse depth would indicate an albedo of $A_{\mathrm{g} \text {,hom }}=0.385_{-0.061}^{+0.060}$ and an atmospheric temperature, $T_{\text {hom }}=2064_{-120}^{+105} \mathrm{~K}$. This would require a much higher albedo than is expected for hot-Jupiters and we consider such a result unrealistic. Furthermore, this is inconsistent with the eclipse depths measured at longer wavelengths (e.g. Croll et al. 2011).

In the instant re-radiation limit, there is no simple selfconsistent model that can explain the emission in the TESS band, suggesting that there must be some energy transport towards the night-side of WASP-12b. Fixing the geometric albedo to zero, we find an upper limit on $f$ at $0.556 \pm 0.047$ with a temperature $T=3128 \mathrm{~K}$. We also attempted to perform a joint fit for our TESS data together with Spitzer data from Bell et al. (2019), and find $A_{\mathrm{g}}$ $=0.32 \pm 0.22$ and $T_{\text {day }}=2510 \pm 134 \mathrm{~K}$, which is consistent with the trend between these parameters found by Wong et al. (2020).

The night-side flux $F_{\mathrm{n}}$ is consistent with zero, indicating a large day-night contrast as expected from these ultra-hot Jupiters (e.g. Perna et al. 2012; Komacek et al. 2017). This is supported by the low-energy redistribution efficiency found above.

\subsection{Peak brightness offset and variability}

We find that the peak planetary brightness is shifted towards the evening terminator by $0.049 \pm 0.015$ in phase, which is typically expected from such planets due to equatorial super-rotation (Showman \& Polvani 2011). Bell et al. (2019) measured both a morning and evening offset in the infrared at 3.6 $\mu \mathrm{m}$, measuring $0.091 \pm 0.017$ degrees in 2010 and $-0.038 \pm 0.011$ degrees in $2013^{1}$. Cloud decks could also cause such observed variability but given WASP-12b's derived temperatures, clouds are unlikely to form. Additionally, WASP-12b has been found to be undergoing orbital decay (Yee et al. 2020) and possibly mass-loss (Bell et al. 2019), which may also play a role in observed variability. However, we observe no significant difference between either TESS orbit (see Appendix A).

The secondary eclipses measured for each orbit are consistent within their uncertainties, and so we can not claim any variability. This may be due to the timescales involved. While we can't directly compare our eclipse depths with previous studies as they use different bandpasses, our results lie between the two observed eclipse depths in Hooton et al. (2019), and are consistent at $2 \sigma$.

\section{CONCLUSIONS}

We present the full optical phase curve of WASP-12b as observed by TESS during year 2 of its mission. With effective detrending and modelling, we are able to derive atmospheric temperatures as well as geometric albedos for limiting cases with regards to heat/energy distribution throughout the planet's atmosphere. We find our results to be consistent with previous measurements, with WASP-12b having a very low albedo and its peak brightness offset towards the evening terminator. We also find that the series is better fit when using an ephemeris model that incorporates the orbital decay outlined in Yee et al. (2020). Lastly, we find no discernible evidence of variability in either of the TESS orbit bins, but future optical data on WASP-12b taken over longer timescales could reveal further evidence of this.

\section{ACKNOWLEDGEMENTS}

NO and CAW acknowledge support from Science and Technology Facilities Council (STFC) grants ST/T506369/1 and ST/P000312/1, respectively. M.J.H. acknowledges the support of the Swiss National Fund under grant 200020_172746. This paper includes data collected by the TESS mission. This paper includes data collected with the TESS mission, obtained from the MAST data archive at the Space Telescope Science Institute (STScI). Funding for the TESS mission is provided by the NASA Explorer Program. STScI

1 Bell et al. (2019) uses a different convention and measure their offsets after eclipse. We have converted their values to our phase and sign convention. 


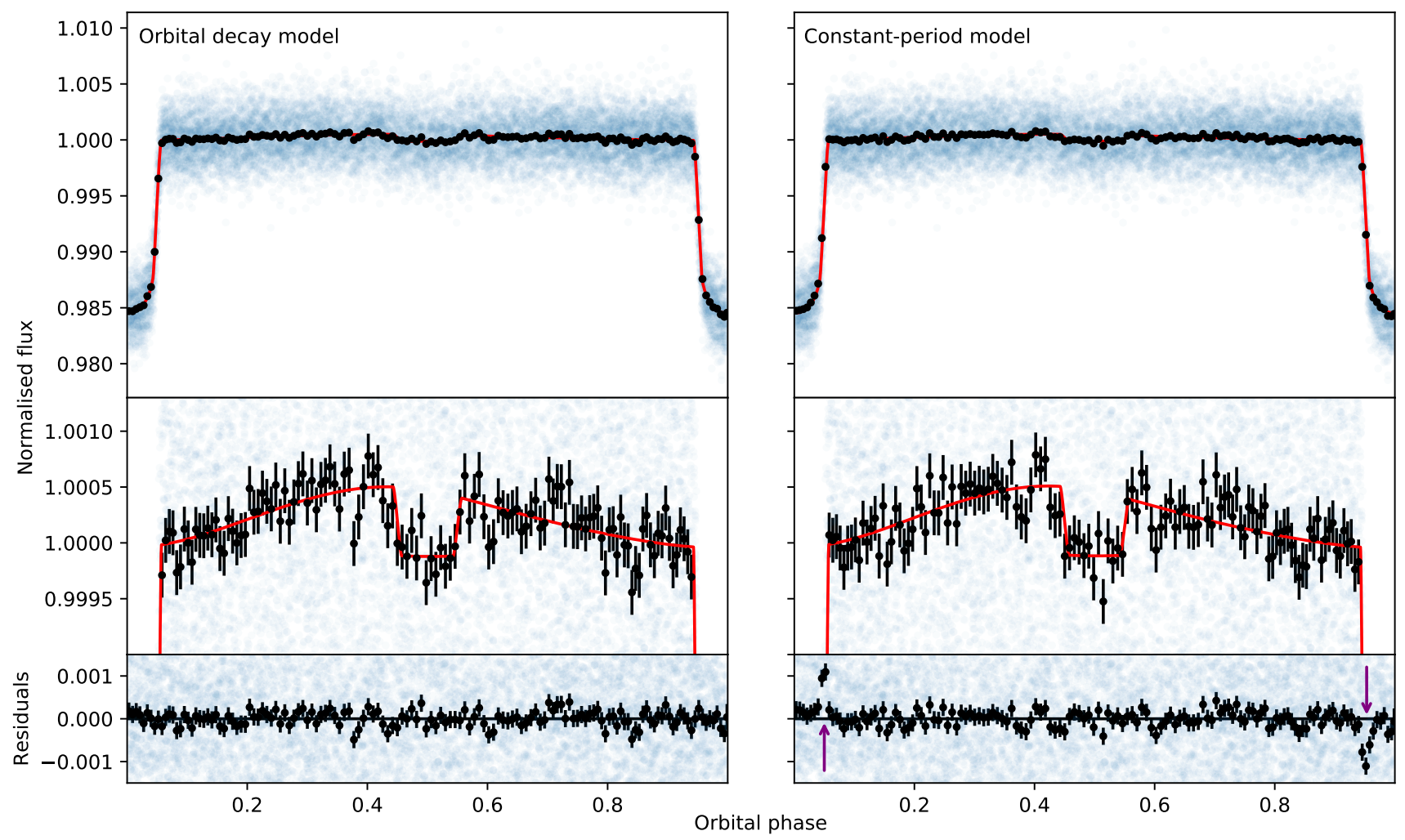

Figure 2. The phase curve of WASP-12b as observed by TESS. Unbinned data plotted in blue, and points in black represent 100-point bins and associated uncertanties. Left: The decaying ephermeris model used here is outlined by Yee et al. (2020). Right: Using a non-decaying, constant-period orbit produces very notable artefacts (purple arrows) in the residuals. The phase curve is best fit with a decaying ephemeris, strongly indicating that WASP-12b's orbit is decaying.

is operated by the Association of Universities for Research in Astronomy, Inc., under NASA contract NAS 5-26555. We also acknowledge use of the following Python packages: SciPy (Virtanen et al. 2020), NumPy (Harris et al. 2020), emcee (Foreman-Mackey et al. 2013), Lightkurve (Lightkurve Collaboration et al. 2018), batman (Kreidberg 2015), Matplotlib (Hunter 2007), AstroPy (Astropy Collaboration et al. 2018).

\section{DATA AVAILABILITY}

The datasets used in this Letter are publicly available from the Mikulski Archive for Space Telescopes (MAST): https://archive.stsci.edu/.

\section{REFERENCES}

Armstrong D. J., de Mooij E., Barstow J., Osborn H. P., Blake J., Saniee N. F., 2016, Nature Astronomy, 1, 0004

Astropy Collaboration et al., 2018, aj, 156, 123

Baraffe I., Homeier D., Allard F., Chabrier G., 2015, A\&A, 577, A42

Bell T. J., et al., 2017, ApJ, 847, L2

Bell T. J., et al., 2019, MNRAS, 489, 1995

Bloemen S., et al., 2011, MNRAS, 410, 1787

Campo C. J., et al., 2011, ApJ, 727, 125

Claret A., 2017, A\&A, 600, A30

Collins K. A., Kielkopf J. F., Stassun K. G., 2017, AJ, 153, 78

Cowan N. B., Machalek P., Croll B., Shekhtman L. M., Burrows A., Deming

D., Greene T., Hora J. L., 2012, ApJ, 747, 82
Croll B., Lafreniere D., Albert L., Jayawardhana R., Fortney J. J., Murray N., 2011, AJ, 141, 30

Crossfield I. J. M., Barman T., Hansen B. M. S., Tanaka I., Kodama T., 2012, ApJ, 760, 140

Delgado Mena E., et al., 2015, A\&A, 576, A69

Esteves L. J., De Mooij E. J. W., Jayawardhana R., 2013, ApJ, 772, 51

Esteves L. J., De Mooij E. J. W., Jayawardhana R., 2015, ApJ, 804, 150

Föhring D., Dhillon V. S., Madhusudhan N., Marsh T. R., Copperwheat C. M., Littlefair S. P., Wilson R. W., 2013, MNRAS, 435, 2268

Foreman-Mackey D., Hogg D. W., Lang D., Goodman J., 2013, PASP, 125, 306

Harris C. R., et al., 2020, Nature, 585, 357-362

Hebb L., et al., 2009, ApJ, 693, 1920

Hooton M. J., de Mooij E. J. W., Watson C. A., Gibson N. P., Galindo-Guil F. J., Clavero R., Merritt S. R., 2019, MNRAS, 486, 2397

Hunter J. D., 2007, Computing in Science \& Engineering, 9, 90

Jenkins J. M., et al., 2016, in Software and Cyberinfrastructure for Astronomy IV. p. 99133E, doi:10.1117/12.2233418

Kipping D. M., 2013, MNRAS, 435, 2152

Knutson H. A., et al., 2007, Nature, 447, 183

Komacek T. D., Showman A. P., Tan X., 2017, ApJ, 835, 198

Kreidberg L., 2015, PASP, 127, 1161

Lightkurve Collaboration et al., 2018, Lightkurve: Kepler and TESS time series analysis in Python, Astrophysics Source Code Library (ascl:1812.013)

Loeb A., Gaudi B. S., 2003, ApJ, 588, L117

López-Morales M., Seager S., 2007, ApJ, 667, L191

López-Morales M., Coughlin J. L., Sing D. K., Burrows A., Apai D., Rogers J. C., Spiegel D. S., Adams E. R., 2010, ApJ, 716, L36

Mandel K., Agol E., 2002, ApJ, 580, L171

Perna R., Heng K., Pont F., 2012, ApJ, 751, 59 


\section{$6 \quad$ N. Owens et al.}

Showman A. P., Polvani L. M., 2011, ApJ, 738, 71

Snellen I. A. G., de Mooij E. J. W., Albrecht S., 2009, Nature, 459, 543

Stevenson K. B., Bean J. L., Madhusudhan N., Harrington J., 2014, ApJ,

791,36

Turner J. D., Ridden-Harper A., Jayawardhana R., 2020, arXiv e-prints, p. arXiv:2012.02211

Virtanen P., et al., 2020, Nature Methods, 17, 26

Wong I., et al., 2020, AJ, 160, 155

Yee S. W., et al., 2020, ApJ, 888, L5

Zellem R. T., et al., 2014, ApJ, 790, 53

Zhang M., et al., 2018, AJ, 155, 83

Zhao M., Monnier J. D., Swain M. R., Barman T., Hinkley S., 2012, ApJ,

744, 122

de Mooij E. J. W., Snellen I. A. G., 2009, A\&A, 493, L35

von Essen C., et al., 2019, A\&A, 628, A115 
APPENDIX A: POLYNOMIAL FITTING AND PDCSAP

For completeness, we jointly fit the phase curve model with an 11th order polynomial for TESS Orbit 1 (TO1) and a 5th order polynomial for TESS Orbit 2 (TO2), using a similar strategy as outlined in in Sect. 3.1 of the form:

$P_{n}(x)=\sum_{k=0}^{n} a_{k} x^{k}$

while sharing parameters specific to the stellar-planetary system. The polynomial order was found by calculating and minimising the Bayesian Information Criterion (BIC) for TO1 and TO2 independently of one another. However, the system parameters derived from each fit differed significantly between both orbits, suggesting that the systematics present are on shorter time-scales than initially thought. Higher order fits prove fruitless as any further polynomial terms could risk fitting and jeopardising the derived phase curve parameters. As a result of these discrepancies, we opt not to use these polynomial fits in deriving our final planetary parameters.

We also include here the individual fits for each TESS orbit using CBVs to detrend the series. We find no significant variability among the binned orbits; however, it must be noted that by binning over each orbit, any variations on short time-scales will be averaged out. A search for variability would require multiple follow-ups of the same system in the same bandpass at significantly different times. Simultaneous observations of WASP-12b could also help rule out the effect of systematics on the observed eclipse depth. We also present here fits for the PDCSAP flux which has been thoroughly detrended using SPOC algorithms. We find the results of these fits to be compatible with the main fit performed in Section 3. Table A1 show our best fit parameters for each dataset and fit.

\section{APPENDIX B: ADDITIONAL PLOTS}

We also present here the corner plot for our fit in Section 3 as well as Figure 2 fixated on the transit to better identify the quality of the different ephemeris fits.

This paper has been typeset from a $\mathrm{T}_{\mathrm{E}} \mathrm{X} / \mathrm{L} \mathrm{T} \mathrm{T}_{\mathrm{E}} \mathrm{X}$ file prepared by the author. 


\section{$8 \quad$ N. Owens et al.}

Table A1. Best-fit parameters for WASP-12b for PDCSAP flux, and SAP flux corrected via polynomial fitting. These parameters were found using an MCMC analysis similar to the approach used in Section 3

\begin{tabular}{|c|c|c|c|c|c|}
\hline Parameter & Prior & PDCSAP best-fit & Polynomial best-fit & TO1 (CBV) & $\mathrm{TO} 2(\mathrm{CBV})$ \\
\hline \multicolumn{6}{|c|}{ Orbital parameters } \\
\hline$P$ (days) & fixed & \multicolumn{4}{|c|}{1.091420107} \\
\hline$t_{0}(\mathrm{BJD})$ & fixed & \multicolumn{4}{|c|}{2456305.455809} \\
\hline$d P / d N$ & fixed & \multicolumn{4}{|c|}{$-10.04 \times 10^{-10}$} \\
\hline$a / R_{*}$ & $\mathcal{N}(3.03,0.034)$ & $3.038 \pm 0.023$ & $3.036 \pm 0.024$ & $3.047 \pm 0.024$ & $3.033_{-0.024}^{+0.023}$ \\
\hline$i$ (degrees) & $\mathcal{N}(83.37,0.7)$ & $83.25_{-0.48}^{+0.50}$ & $83.22_{-0.51}^{+0.55}$ & $83.19_{-0.50}^{+0.53}$ & $83.37_{-0.51}^{+0.53}$ \\
\hline \multicolumn{6}{|c|}{ Transit parameters } \\
\hline$R_{\mathrm{p}} / R_{*}$ & - & $0.1173 \pm 0.0007$ & $0.1171 \pm 0.0008$ & $0.1181 \pm 0.0009$ & $0.1166 \pm 0.0009$ \\
\hline$u_{1}$ & - & $0.212_{-0.114}^{+0.159}$ & $0.162_{-0.106}^{+0.165}$ & $0.314_{-0.176}^{+0.259}$ & $0.146_{-0.102}^{+0.173}$ \\
\hline$u_{2}$ & - & $0.301_{-0.132}^{+0.179}$ & $0.374_{-0.135}^{+0.190}$ & $0.146_{-0.261}^{+0.178}$ & $0.405_{-0.137}^{+0.203}$ \\
\hline \multicolumn{6}{|c|}{ Phase curve } \\
\hline$F_{\mathrm{ecl}}(\mathrm{ppm})$ & - & $577_{-72}^{+71}$ & $494_{-83}^{+81}$ & $655_{-98}^{+96}$ & $553 \pm 110$ \\
\hline$A_{\mathrm{p}}(\mathrm{ppm})$ & - & $530 \pm 60$ & $537 \pm 73$ & $530_{-82}^{+80}$ & $563_{-94}^{+92}$ \\
\hline$\theta$ (phase) & - & $0.061 \pm 0.015$ & $-0.002 \pm 0.017$ & $0.063 \pm 0.022$ & $0.034 \pm 0.021$ \\
\hline$M_{\mathrm{p}}\left(\mathrm{M}_{\mathrm{J}}\right)$ & $\mathcal{N}(1.47,0.076)$ & $1.48 \pm 0.08$ & $1.48 \pm 0.08$ & $1.48 \pm 0.08$ & $1.47 \pm 0.08$ \\
\hline$F_{\mathrm{n}}(\mathrm{ppm})$ & - & $47 \pm 94$ & $-43 \pm 111$ & $125_{-128}^{+125}$ & $-10_{-145}^{+144}$ \\
\hline
\end{tabular}




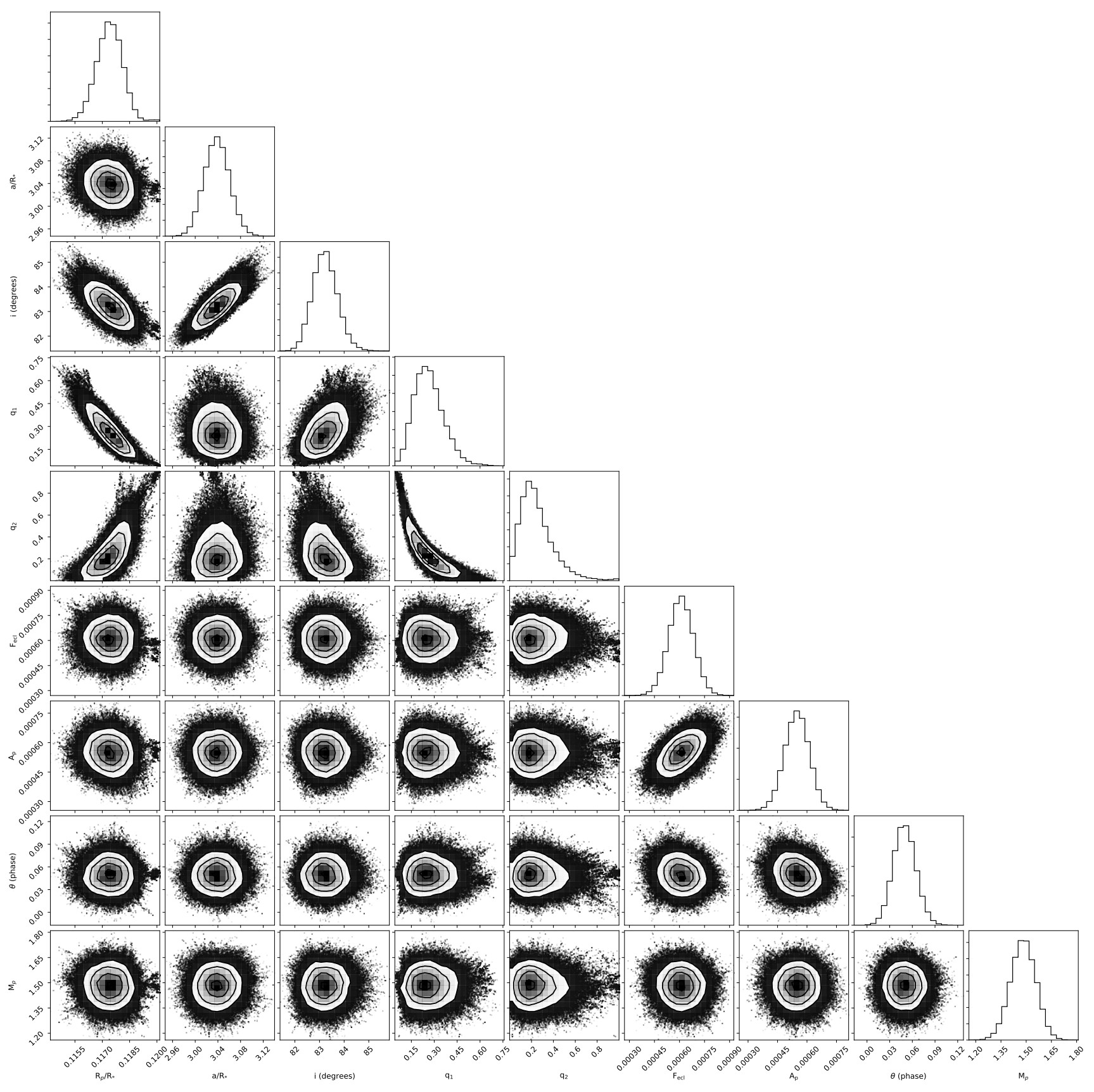

Figure B1. Reduced size corner plot from our analysis in Section 3. 


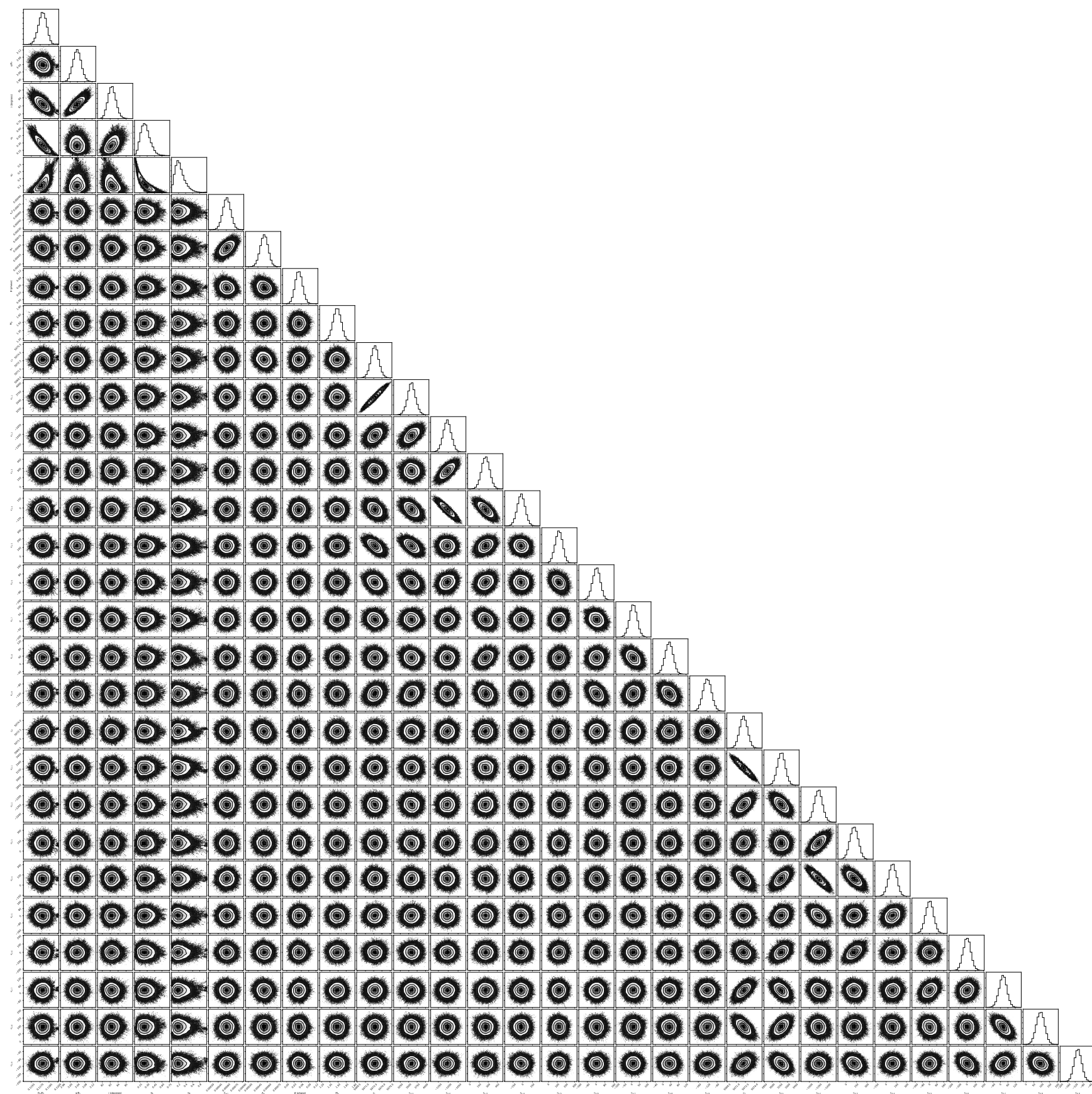

Figure B2. Full corner plot from our analysis in Section 3, including coefficients for CBVs (included to show that CBV coefficients are not degenerate with planetary/transit parameters). 

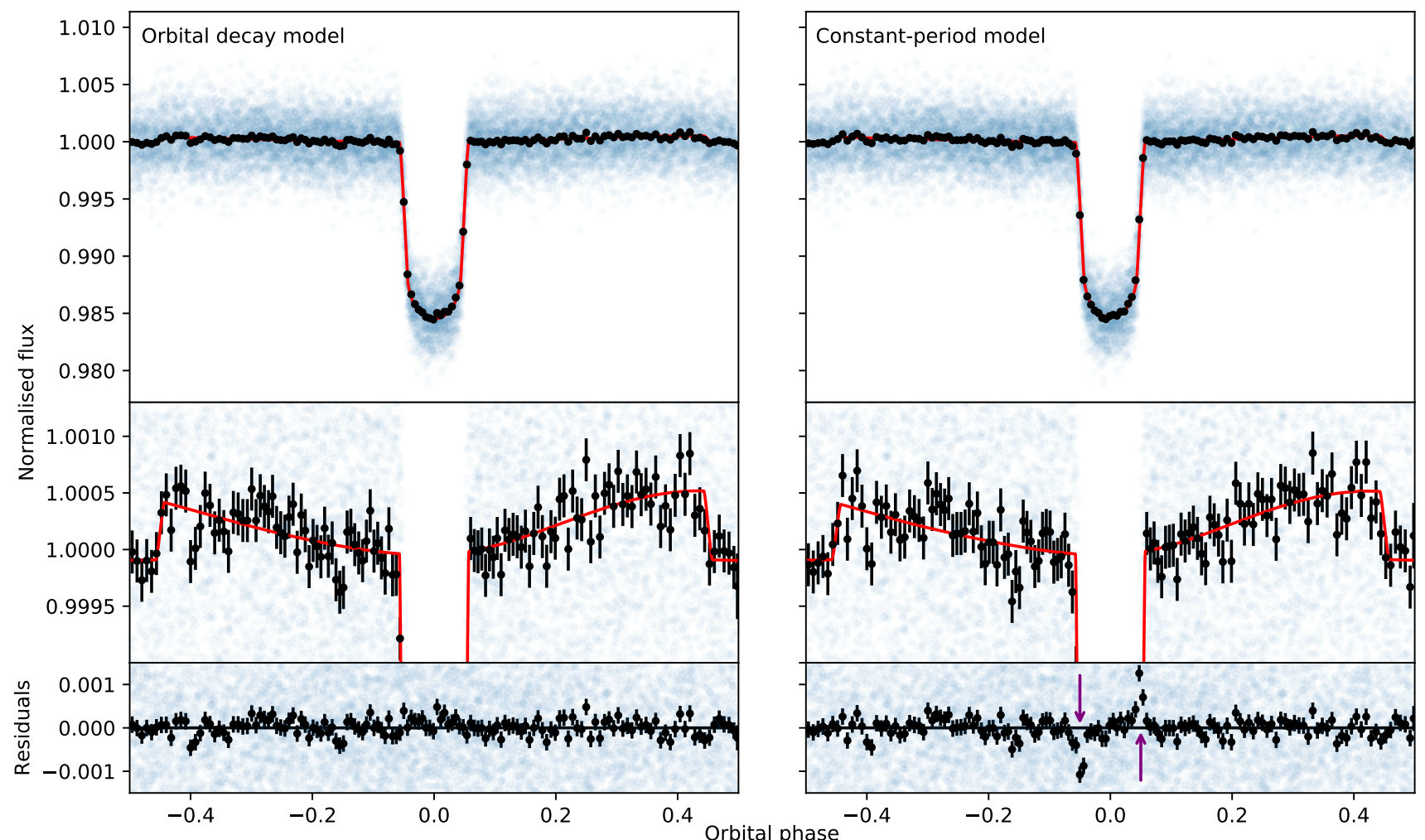

Figure B3. Same as Figure 2, but centred on the transit at $\phi=0$. Residual artefacts are clearly present in the constant-period model plot. 